\title{
Universality in the firing of minicolumnar-type neural networks
}

\author{
Kanders, Karlis ; Stoop, Norbert ; Stoop, Ruedi
}

\begin{abstract}
An open question in biological neural networks is whether changes in firing modalities are mainly an individual network property or whether networks follow a joint pathway. For the early developmental period, our study focusing on a simple network class of excitatory and inhibitory neurons suggests the following answer: Networks with considerable variation of topology and dynamical parameters follow a universal firing paradigm that evolves as the overall connectivity strength and firing level increase, as seen in the process of network maturation. A simple macroscopic model reproduces the main features of the paradigm as a result of the competition between the fundamental dynamical system notions of synchronization vs chaos and explains why in simulations the paradigm is robust regarding differences in network topology and largely independent from the neuron model used. The presented findings reflect the first dozen days of dissociated neuronal in vitro cultures (upon following the developmental period bears similarly universal features but is characterized by the processes of neuronal facilitation and depression that do not require to be considered for the first developmental period). A key element for explaining processes in nature by physics has been the art of choosing the optimal level of description for the effects to be described. In our current challenge to explain important aspects of our brain by means of physics, we still largely miss such a handle at many levels: To what detail, e.g., is it necessary to model neurons and their connectivity to understand what their neural network is doing? For simple small-size networks of minicolumnar type (by many considered as a potential module underlying the function of the cortex), we show that all networks from this large network class follow the same- universal-behavior, as their overall connectivity strength is enhanced. Moreover, the paradigm that they follow can be explained in terms of low-dimensional dynamical systems theory, which reveals the origin of the universal behavior. Our findings suggest that other network classes could be treated in a similar manner. The uncovered universality permits us to substantially limit the degree of details required to model cortical computation, which opens up a novel perspective toward more effective simulations of and investigations into close-tobiology neural networks and sheds a novel perspective on biological multiscale information processing. From the practical side, our findings imply that biological neural networks with strong parallels to the increase of a connectivity strength will develop closely along the uncovered paradigm. Examples are neuronal cultures at the early stage of their development or biochemical processes that globally enhance the connectivity strength among the elements of the neural network.
\end{abstract}

DOI: https://doi.org/10.1063/1.5111867

Posted at the Zurich Open Repository and Archive, University of Zurich

ZORA URL: https://doi.org/10.5167/uzh-184153

Journal Article

Published Version

Originally published at: 
Kanders, Karlis; Stoop, Norbert; Stoop, Ruedi (2019). Universality in the firing of minicolumnar-type neural networks. Chaos (Woodbury, N.Y.), 29(9):093109.

DOI: https://doi.org/10.1063/1.5111867 


\section{Universality in the firing of minicolumnar- type neural networks}

Cite as: Chaos 29, 093109 (2019); https://doi.org/10.1063/1.5111867

Submitted: 30 May 2019. Accepted: 19 August 2019. Published Online: 09 September 2019

Karlis Kanders, Norbert Stoop, and Ruedi Stoop

\section{COLLECTIONS}

F This paper was selected as Featured
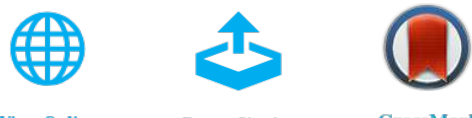

\section{ARTICLES YOU MAY BE INTERESTED IN}

Feed-forward artificial neural network provides data-driven inference of functional connectivity

Chaos: An Interdisciplinary Journal of Nonlinear Science 29, 091101 (2019); https:// doi.org/10.1063/1.5117263

Percept-related EEG classification using machine learning approach and features of functional brain connectivity

Chaos: An Interdisciplinary Journal of Nonlinear Science 29, 093110 (2019); https://

doi.org/10.1063/1.5113844

Reconstructing dynamical networks via feature ranking

Chaos: An Interdisciplinary Journal of Nonlinear Science 29, 093107 (2019); https://

doi.org/10.1063/1.5092170

\section{Scilight}




\title{
Universality in the firing of minicolumnar-type neural networks
}

\author{
Cite as: Chaos 29, 093109 (2019); doi: 10.1063/1.5111867 \\ Submitted: 30 May 2019. Accepted: 19 August 2019. \\ Published Online: 9 September 2019

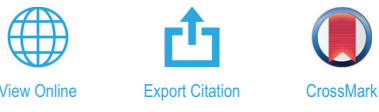

Karlis Kanders, ${ }^{1,2}$ Norbert Stoop, ${ }^{2,3}$ and Ruedi Stoop ${ }^{1,2, a)}$

\begin{abstract}
AFFILIATIONS
${ }^{1}$ Institute of Neuroinformatics, University of Zurich and ETH Zurich, 8057 Zurich, Switzerland

${ }^{2}$ Institute for Building Materials, ETH Zurich, 8092 Zurich, Switzerland

${ }^{3}$ Department of Mathematics, Massachusetts Institute of Technology, Cambridge, Massachusetts 02139-4307, USA
\end{abstract}

${ }^{a}$ Author to whom correspondence should be addressed: ruedi@ini.phys.ethz.ch

\begin{abstract}
An open question in biological neural networks is whether changes in firing modalities are mainly an individual network property or whether networks follow a joint pathway. For the early developmental period, our study focusing on a simple network class of excitatory and inhibitory neurons suggests the following answer: Networks with considerable variation of topology and dynamical parameters follow a universal firing paradigm that evolves as the overall connectivity strength and firing level increase, as seen in the process of network maturation. A simple macroscopic model reproduces the main features of the paradigm as a result of the competition between the fundamental dynamical system notions of synchronization vs chaos and explains why in simulations the paradigm is robust regarding differences in network topology and largely independent from the neuron model used. The presented findings reflect the first dozen days of dissociated neuronal in vitro cultures (upon following the developmental period bears similarly universal features but is characterized by the processes of neuronal facilitation and depression that do not require to be considered for the first developmental period).
\end{abstract}

Published under license by AIP Publishing. https://doi.org/10.1063/1.5111867

A key element for explaining processes in nature by physics has been the art of choosing the optimal level of description for the effects to be described. In our current challenge to explain important aspects of our brain by means of physics, we still largely miss such a handle at many levels: To what detail, e.g., is it necessary to model neurons and their connectivity to understand what their neural network is doing? For simple smallsize networks of minicolumnar type (by many considered as a potential module underlying the function of the cortex), we show that all networks from this large network class follow the same-universal-behavior, as their overall connectivity strength is enhanced. Moreover, the paradigm that they follow can be explained in terms of low-dimensional dynamical systems theory, which reveals the origin of the universal behavior. Our findings suggest that other network classes could be treated in a similar manner. The uncovered universality permits us to substantially limit the degree of details required to model cortical computation, which opens up a novel perspective toward more effective simulations of and investigations into close-to-biology neural networks and sheds a novel perspective on biological multiscale information processing. From the practical side, our findings imply that biological neural networks with strong parallels to the increase of a connectivity strength will develop closely along the uncovered paradigm. Examples are neuronal cultures at the early stage of their development or biochemical processes that globally enhance the connectivity strength among the elements of the neural network.

\section{INTRODUCTION}

A central quest in complexity science is to understand how topology influences the overall behavior of networks. Brain dynamics is one example where a huge synaptic connectivity corpus has been gathered in the hope to better understand how human thoughts and actions are generated. While general topological features that may bear influence on network dynamics have been identified, ${ }^{1-3}$ for many fundamental functional properties, such as orientation selectivity in the visual cortex, ${ }^{4}$ optimal computational capacity, ${ }^{5}$ and precise spiking patterns in small invertebrate circuits, ${ }^{6}$ the detailed network connectivity, however, has been shown to be not essential. Conversely, a fixed structural connectivity can host a vast range of distinct network dynamics depending on input, physiological, and biochemical parameters. ${ }^{78}$ From these, various control pathways select particular 
patterns. A particular important one is neuromodulation, where by means of emitted chemicals (neurotransmitters), ${ }^{9,10}$ whole populations of neurons are up- or down-regulated. A clear picture highlighting to what extent neuronal firing modality can be attributed to topological connectivity vs neuromodulation ${ }^{11,12}$ is, however, still missing.

We investigate this question by studying the firing dynamics of close-to-biology neural networks for a considerable range of network topologies. In these networks, we monitor the global amount of synaptic efficacy, mimicking the effect of a neuromodulator, or, seen on a much larger temporal scale, the maturation of developing neural networks (increase of synaptic strengths is a characteristic of network maturation). Using a multifaceted characterization of network firing, we find that upon an increase of the synaptic connectivity strength, the behavior of networks evolves in a unique way essentially independent from network topology. To understand the origin of this phenomenon, we introduce a macroscopic, meanfieldlike model that rationalizes our findings in terms of basic dynamical systems theory. Finally, we provide experimental support for our findings from developing dissociated neuron cultures.

\section{A CLOSE-TO-BIOLOGY MODEL OF MINICOLUMNAR-TYPE NEURAL NETWORKS}

Our simulation approach is technically based on a previously introduced description of the cortical minicolumn., ${ }^{5,13}$ This approach that applies to a very general class of neural networks allows for a separation of the input into many small randomlike and fewer more strongly connected synchronized inputs. In a cortical minicolumn (generally composed of 80-140 neurons ${ }^{14}$ ) from the many synaptic contacts impinging on a neuron (order of $10^{4}$ ), a high number originate from the outside of the column, but specific physiological experiments suggest that columnar dynamics is strongly dominated by input roughly across a columnar dimension. ${ }^{15,16}$ This, because for spike generation, of the order of $10^{2}$ synapses need to activate in a short time window. By uncoordinated input from all the area external to the column, this is hardly feasible, in contrast to strongly temporally coordinated synaptic input from a few strongly connected neighboring neurons that may even enhance their effect by phase-locking (although this may be obscured by external activity modulation across a midterm temporal scale). Despite great efforts, the exact biological architecture and the functional role of minicolumns have remained elusive (not so surprisingly, if they indeed have the role of abstract computational units). Irrespective of the outcome of the corresponding dispute, our results presented below apply to a large class of neural networks that can be described in the following way. Synaptic input between strongly connected neurons can be concatenated into a mesoscopic synapse. To represent incoherent inputs, a random signal is added to this synaptic input; to estimate the effect by coherent input from outside of the column, autonomously chaotically firing neurons are embedded into the network structure (details below). For reasons of genericity, our presentation will emphasize on random (RA) network architecture, but we also check then consistency of our results on small-world (SW) and on $3 \mathrm{D}$ columnar topology (CL) networks. The latter networks have been claimed to specifically reflect biological minicolumnar connectivity (supplementary material). Our simulation results are mostly based on networks with $N=128$ neurons, of which $N_{\mathrm{ex}}=102$ are of excitatory and $N_{\text {inh }}=26$ of inhibitory type (using their well-known $4: 1 \mathrm{ratio}^{17}$ and following the example of a minicolumn (checks on networks of quartered or quadrupled size are included where of interest). We will show that all of these networks follow the same firing paradigm if their connectivity strength is globally upregulated.

The connectivity of neurons is directional, with an average probability of 0.04 for each topology considered (supplementary material). Synaptic upregulation was modeled by scaling the strength of synaptic connections between neurons by a global "scaling factor." Neuronal dynamics was established by Rulkov neurons, ${ }^{18,19}$ which reproduce, at low computational costs, a huge range of the experimentally observed aspects of neuronal firing behavior. ${ }^{19,20}$ Their dynamics is defined by the two-dimensional iterative map,

$$
\begin{aligned}
& x_{n+1}^{(i)}= \begin{cases}\frac{\psi}{1-x_{n}^{(i)}}+u_{n}^{(i)} & x_{n}^{(i)} \leq 0, \\
\psi+u_{n}^{(i)} & 0<x_{n}^{(i)}<\psi+u_{n}^{(i)} \wedge x_{n-1}^{(i)} \leq 0, \\
-1 & x_{n}^{(i)} \geq \psi+u_{n}^{(i)} \vee x_{n-1}^{(i)}>0,\end{cases} \\
& y_{n+1}^{(i)}=y_{n}^{(i)}-\mu\left(1+x_{n}^{(i)}\right)+\mu\left(\sigma+I_{n}^{(i)}\right),
\end{aligned}
$$

where $n$ is the iteration step, $x_{n}^{(i)}$ is the membrane potential of the $i$ th neuron, $y_{n}^{(i)}$ describes a regulatory subsystem able to turn firing on and off (Fig. 1 in the supplementary material), and $\psi, \mu, \beta$, and $\sigma$ are scalar parameters. $u_{n}^{(i)}=y_{n}^{(i)}+\beta I_{n}^{(i)}$, where $I_{n}^{(i)}$ describes the synaptic input to neuron $i .{ }^{19}$ Neuronal spikes are modeled by the firing variable $\xi_{n+1}^{(j)}=1,0<x_{n}^{(j)}<\psi+u_{n}^{(j)} \wedge x_{n-1}^{(j)} \leq 0 ; 0$ otherwise. In this way, $\xi_{n+1}^{(j)}$ carries a value 1 (a spike) if $x_{n}^{(j)}$ attains the maximum value of the map at iteration $n$ and a value 0 (no spike) otherwise. Spikes are transmitted along synapses; spike-generated currents at synapses are characterized by a steplike increase upon an incident event, followed by an exponential decay in time, which can be expressed as

$$
\begin{aligned}
I_{n+1}^{(i)}= & \eta I_{n}^{(i)}+W\left(\sum_{j=1}^{N_{\mathrm{ex}}} w_{i j}\left[x_{\mathrm{rp}}^{\mathrm{ex}}-x_{n}^{(i)}\right] \xi_{n}^{(j)}\right. \\
& \left.+\sum_{j=N_{\mathrm{ex}}+1}^{N} w_{i j}\left[x_{\mathrm{rp}}^{\mathrm{inh}}-x_{n}^{(i)}\right] \xi_{n}^{(j)}+w_{\mathrm{ext}}\left[x_{\mathrm{rp}}^{\mathrm{ex}}-x_{n}^{(i)}\right] \xi_{n}^{\operatorname{ext}(i)}\right) .
\end{aligned}
$$

In this equation, $\eta<1$ is the decay rate, and $x_{\mathrm{rp}}^{\mathrm{ex}}=0.0$ and $x_{\mathrm{rp}}^{\mathrm{inh}}=-1.1$ are the reversal potentials of excitatory and inhibitory synapses, respectively. Synaptic connectivity between neurons was described by the connectivity matrix $w_{i j}$ with $w_{i j}=w_{\mathrm{ex}}\left(w_{i j}=w_{\text {inh }}\right)$ if neuron $i$ is the terminal of an excitatory (inhibitory) synaptic connection from neuron $j$ and $w_{i j}=0$ otherwise. The term $\sim w_{\text {ext }}$ accounts for "dynamical randomness" (e.g., synaptic noise from spontaneous vesicle release $\left.{ }^{21}\right)$. The corresponding noisy spike trains are represented by the spike variable $\xi_{n}^{\operatorname{ext}(i)}$ that assumes the values $\xi_{n+1}^{\operatorname{ext}(i)}=1$, $p<p^{\text {ext }}$, and 0 otherwise. At each iteration, $p$ is drawn with uniform probability in $(0,1)$. We choose $p^{\text {ext }}=6 \cdot 10^{-4}$ to render this input temporally sparse. Parameter $W$ is a connectivity-scaling factor that mimicks the action of neuromodulators by uniformly affecting synaptic strength in the network.

With neural parameters chosen as $\psi=3.6, \mu=0.001$, $\beta=0.133, \quad \sigma=0.09, \quad w_{\mathrm{ex}}=w_{\mathrm{ext}}=0.6, \quad w_{\mathrm{inh}}=1.8$ throughout, 
isolated Rulkov neurons $\left(I_{n} \equiv 0\right)$ are below firing threshold and undergo a subcritical Neimark-Sacker bifurcation from silence into firing as $\sigma+I_{n} \gtrsim 0.1$, a property that is associated with Class II neuronal behavior. ${ }^{22}$ One of the excitatory neurons is, however, randomly chosen to be a persistently firing neuron (PN), by raising its value of $\sigma$ to 0.103 , which puts it into a subtly chaotic firing state. Such a neuron may represent coherent external (e.g., sensory) input, reflect biological evidence, ${ }^{23}$ and provide more detailed insight into the internal organization of the network (see below). Intrinsically silent neurons in the network can be pushed into firing if $I_{n}$ joins the effect of $\sigma$, entailing activation due to (1) internal network interaction, (2) excitatory noisy spike-train input $\left[\xi^{\operatorname{ext}(i)}\right]$, and (3) activation by the PN. In this sense, the PN is related to the concept of "leader neurons" ${ }^{24}$ or "nucleation sites." 25

For different network constructions, we simulated the effect of neuromodulation by an increase of parameter $W \in[0,1]$ in Eq. (2). From the neural activity, we computed the full set of Lyapunov exponents (we denote the largest one by $\lambda_{1}$ ), Kolmogorov-Sinai (KS) entropy, $\mathrm{PN}$ and network firing rates, oscillation frequency, and a measure of synchronization ${ }^{26}$ (supplementary material). The used synchronization measure compares the variance of fluctuations of the population-averaged Rulkov vectors $y_{n}^{(i)}$ with the average variance of the individual neurons. This allows us to detect at least zero-lag $1: 1$ phase-lockings, which in practice proved sufficient to determine to what extent the network neurons were spiking in unison. For the experimental data, where we cannot access the neuronal state variables, we employed binned spike count time series of the electrode data.

\section{RESULTS}

Surprisingly, we observe a transition paradigm of neural dynamics that is largely independent of the different network types (Fig. 1; see the supplementary material for additional material). Across hundreds of runs, the paradigm has been found to be exceptionally stable, with only minor changes under resampling of network topologies and Rulkov parameters at each value of $W$, as well as the presence or absence of a PN (supplementary material). The paradigm is characterized by a monotonic increase of $\mathrm{PN}$ and network firing rates with increasing $W>0.12$, a peak in the oscillation frequency around $W \sim 0.3$ occurring slightly after a hump in synchrony, followed by a peak in the KS-entropy. $\lambda_{1}$ was consistently above 0 , indicating a chaotic dynamics for $W>0.12$.

To identify the mechanism behind the paradigm, we focus on random networks with an in-degree constrained to $k=5$ to enable an unbiased comparison of results (except for the demonstration, this constraint is inessential, see below). This value of $k$ equals the mean degree of the unconstrained (random) networks obtained for the chosen connectivity probability (cf. also Ref. 16). In-degree constrained networks can be expected to express the paradigm in its purest form (Fig. 2), as in-degree unconstrained networks occasionally exhibit small shifts of the paradigm along the $W$ axis, which naturally smears out smaller details of the paradigm. After passing an "avalanche critical point" ${ }^{27}$ close to point $A$ in Fig. 2(b), regime (I) (supplementary material), the first network transition occurs. At this stage, the previously dominant PN becomes a part of the network, which is signaled by the onset of a coevolution of the largest
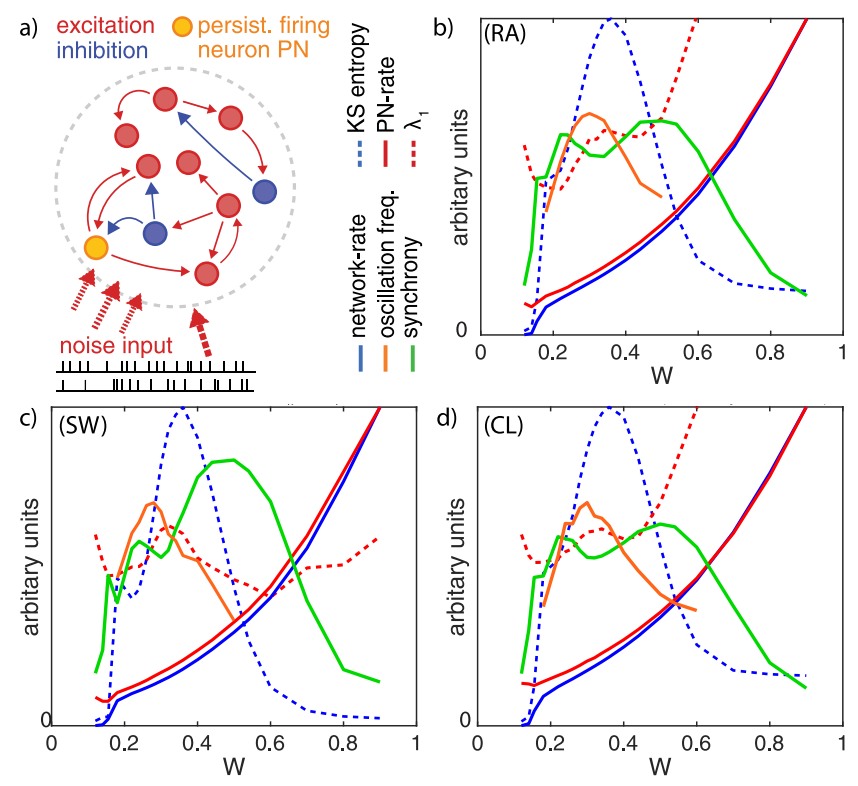

FIG. 1. (a) Schematics of neural networks with external noise and persistently firing neuronal inputs. (b)-(d) Neural network firing dynamics is robust regarding different choices of network topologies: Measures of neural activity follow with increasing synaptic strength $W$ very similar characteristics, showing only small variation between random (b), small-world (c), and columnar (d) network topologies (and almost no variation within groups); displayed results are the averages over 10 realizations, where standard deviations are already sufficiently small. Vertical axes have been rescaled to allow direct comparison of the different measures. For typical axes ranges, cf. Figs. 2 and 7 of the supplementary material.

Lyapunov exponent $\lambda_{1}$ with the KS-entropy and an ever larger number of ever more unstable individual chaotic dynamical (sub)modes (supplementary material). Our analysis also finds that the latter are generated within a "coherent" network behavior, indicated by a noticeable off-set of the largest exponent from the bulk of unstable exponents (supplementary material). Increasing the coupling $W$ starts to synchronize these unstable modes, reducing their instability (some become even frozen) at maintained coherence, until a state of maximal synchronization is achieved (II). Upon further increased coupling, the bounds by synchronization are broken, and a growing number of ever more unstable subnets is created again, ending at maximized submode-instability with the loss of global coherence (III). At even larger coupling, these modes still partially synchronize (IV), at further reduced instability, until the synchronization is fully abolished. At this point, the network's ability to coherently and variably react to a stimulus is lost (no positive Lyapunov exponents remain), which restricts the regime of biological interest to $W \lesssim 0.55$.

\section{MEANFIELDLIKE DYNAMICAL SYSTEM DESCRIPTION OF THE UNIVERSALITY}

Our results thus suggest that the rich paradigms of firing exhibited by close-to-biology neural networks are more systematic than could be expected. To scrutinize the origin of this phenomenon, 
a)

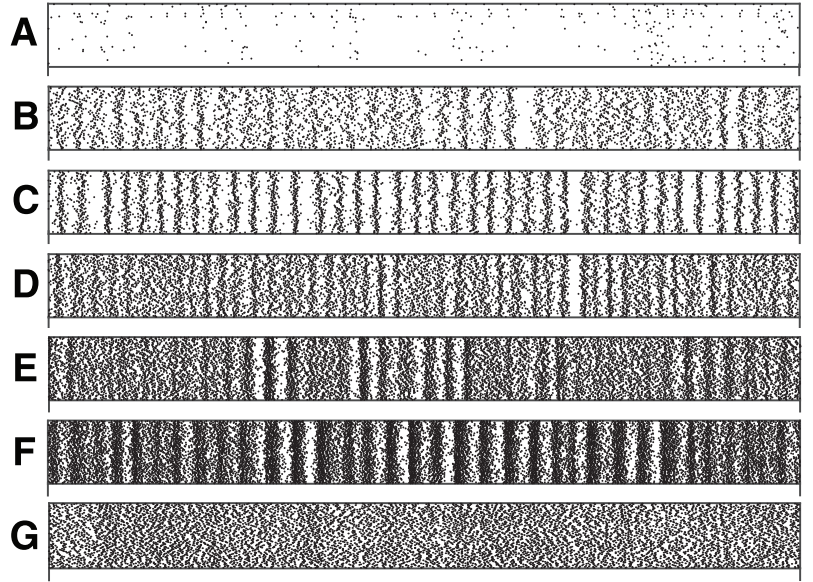

Neuron (1.. 128)

$\longrightarrow$ Timestep $\mathrm{n}$ b)

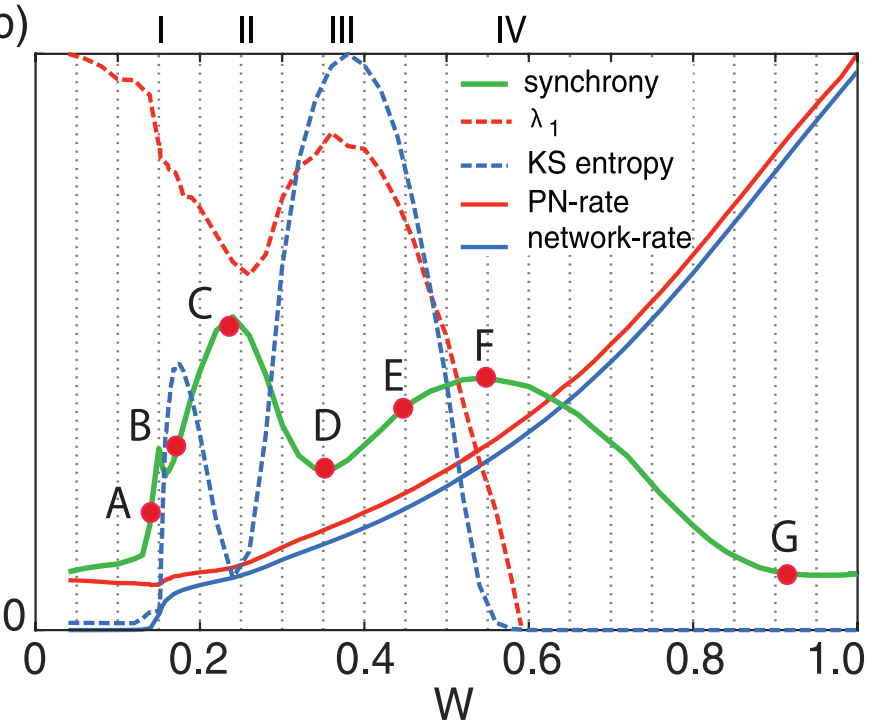

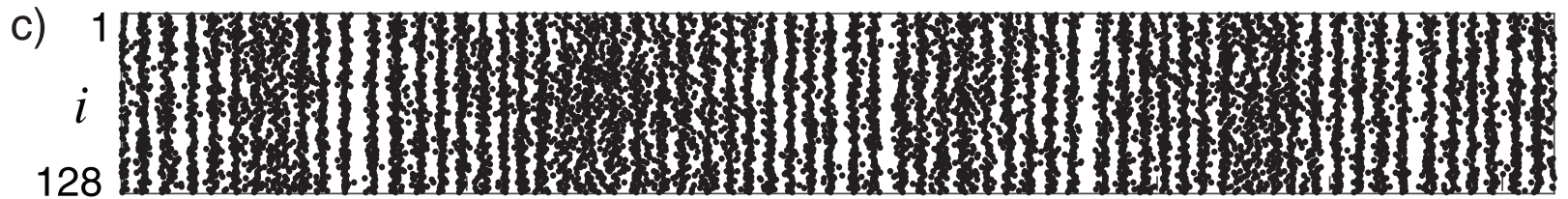
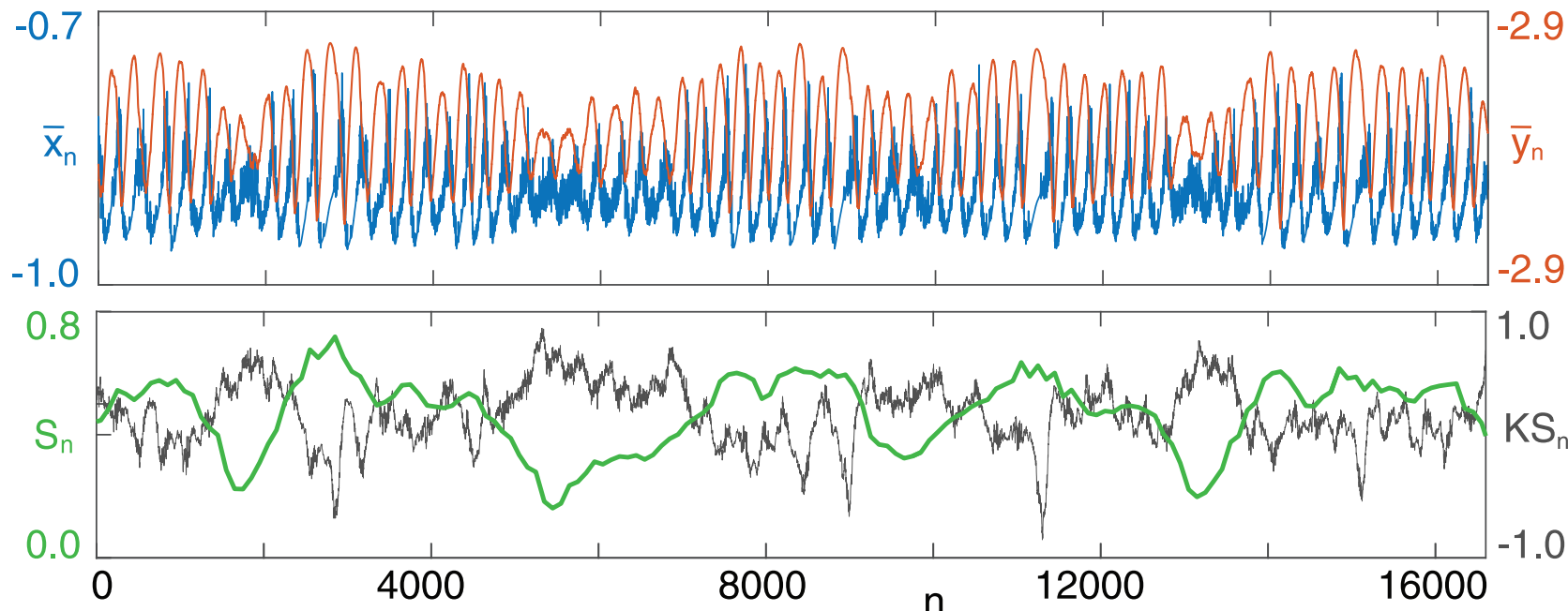

FIG. 2. In-degree-constrained networks $(k=5)$. (a) Raster plots of firing patterns for $W=0.14,0.18,0.24,0.34,0.44,0.54,0.9 ;$ Time steps: $10000(A-F)$ and $2000(G)$. (b) In-degree constraint enhances first peak in synchrony. Red dots indicate corresponding raster plots in (a). (c) Network raster plot slightly after point $C$ ( $W=0.26)$ (top), averaged network variables $\bar{x}$ and $\bar{y}$ (middle). While synchrony $S$ and $K S$-entropy evaluate as time averages, the temporal contributions to these values from the nth time step, $S_{n}$ and $K S_{n}$, are locally antagonistic (bottom), with the negative values of $K S_{n}$ indicating strongly nonhyperbolic dynamics.

we observe that the central part of our transformation paradigm is characterized by the antagonistic influence of synchronization and chaos [Fig. 2(b)]. From a basic theory of low-dimensional dynamical systems, ${ }^{28}$ this is unexpected, but here, the consequence of the large number of active degrees of freedom that gives rise to a simple macroscopic picture explaining the phenomenon is as follows. We describe the network dynamics by averaged neural network variables $\bar{x}_{n}=\frac{1}{N} \sum_{i=1}^{N} x_{n}^{(i)}, \bar{y}_{n}=\frac{1}{N} \sum_{i=1}^{N} y_{n}^{(i)}$. These variables exhibit a rather simple oscillatory dynamics for the in-degree constrained network of Fig. 2(c): Small fluctuations around some nonzero value 
of $x_{n}^{(i)}, y_{n}^{(i)}$ alternate intermittently with large synchronized oscillations (Fig. 2). The time-resolved synchrony $S_{n}$ and KS-entropy $K S_{n}$ (the latter denoting the temporal contribution to $K S$ that, therefore, can consist of a negative value) show an antagonistic behavior. The macroscopic behavior thus resembles a process in which $y_{n}^{(i)}$ describes a "resource," which facilitates the growth of a second process $x_{n}^{(i)}$ that in turn has a negative effect on $y_{n}^{(i)}$. To test this hypothesis, we consider a generic predator-prey dynamics, ${ }^{29}$

$$
\dot{x}=b x p(y)-d x, \quad \dot{y}=\operatorname{ryg}(y)-x p(y),
$$

where $p(y)=\left(y^{3}+y\right) /\left(y^{3}+y+a^{-1}(1+\exp [-y+3])\right)$ is a Hollingtype response ${ }^{29}$ describing the ability of neurons to draft other neurons into firing, and $g(y)=1-y / c$ implements a steady-state of the network resource at $y=c . a, b, c, d$, and $r$ are positive constants and "'” denotes the derivative with respect to time. In a biological interpretation, $x$ can be seen as firing activity that depletes a neurotransmitter resource $y$. The typical situation described by Eq. (3) is characterized by the presence of a stable limit cycle and a central repelling fixed-point. If fluctuations are taken into account, the dynamics of $\{x, y\}$ would linger between these two objects [Fig. 3(a)]. A detailed analysis shows that an increase of $W$ corresponds in Eq. (3) to a simultaneous increase of $a$ and $b$. Keeping $d=0.4, c=4$, and $r=1.2$ fixed, we increase $\{a, b\}$ along the line $b(a)=a / 40+0.1$, $12 \leq a \leq 36$, yielding monotonically increasing amplitudes $x_{A}$ of the macroscopic firing rate $x$ and convex dependence of the leading Lyapunov exponent $\lambda$ and the oscillation frequency $f$, similar to the one observed in the detailed model [Fig. 3(b)]. At the maximal value of $\lambda$ and $f(\{a, b\} \simeq\{20,0.6\})$, the repelling fixed-point $\left\{x^{*}, y^{*}\right\}$ that characterizes the desynchronized network state is minimally unstable. Perturbations that push the state vector into the vicinity of $\left\{x^{*}, y^{*}\right\}$ then take longer to relax back onto the limit cycle. This mechanism leads to the decreased network synchrony and to the pattern oscillation frequency maximum in the shaded region in Fig. 3(b) (supplementary material). Even if the in-degree constraint is removed, the macroscopic model [Eq. (3)] still describes firing changes reasonably well if the slope of $b(a)$ is adapted (supplementary material). The obtained results reveal a collective phenomenon as the origin of the observed universality in the firing behavior.

\section{EXPERIMENTAL SUPPORT}

Neurons in culture establish synaptic connections that in the development over the first weeks become stronger, ${ }^{30}$ suggesting a qualitative correspondence between "days in vitro" (DIV) and the connectivity-scaling factor $W$. Analyzing spike data from dissociated hippocampal neurons of E18 Sprague-Dawley rats (see Ref. 31 for details) over several DIVs, we find that the experimentally obtained synchrony and firing rates follow closely the overall pathway of our paradigm [Fig. 3(c)], although we cannot expect our simple model to reflect all fine-level details of the experimental behavior. In network simulations, the observed $\sim 4$ spikes/1000 steps at $\mathrm{W}=0.2$ and $\sim 16$ spikes $/ 1000$ steps at $W=0.6$ equate to about 8 and $32 \mathrm{~Hz}$, respectively, if one time step is equated to $0.5 \mathrm{~ms}$ (Rulkov's recommendation $\left.{ }^{19}\right)$. Such values are in agreement with in vivo biological data, e.g., cat visual cortex V1. ${ }^{32}$ In neuronal cultures such as ours, firing rates have, however, been found to substantially depend
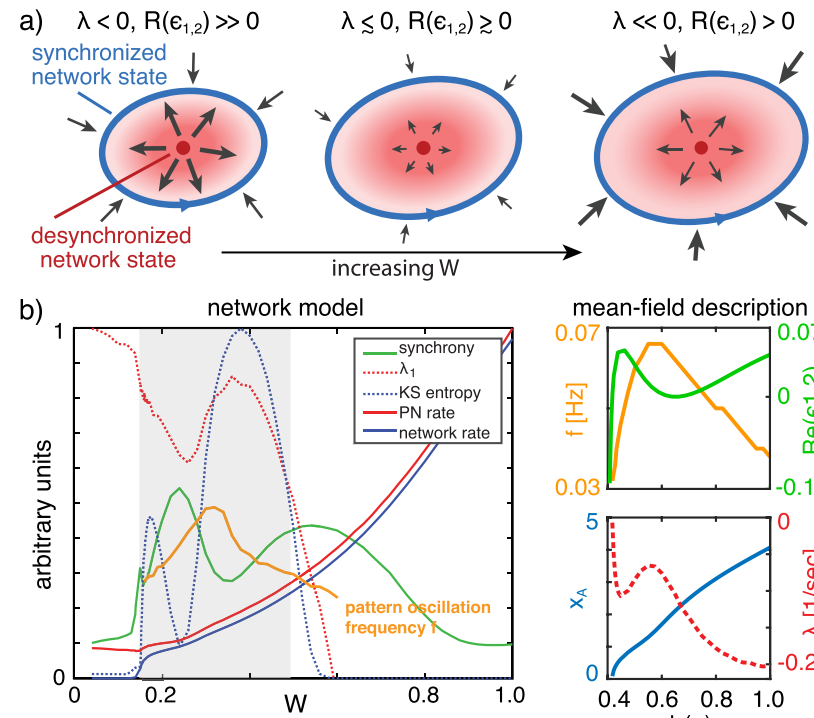

mean-field description
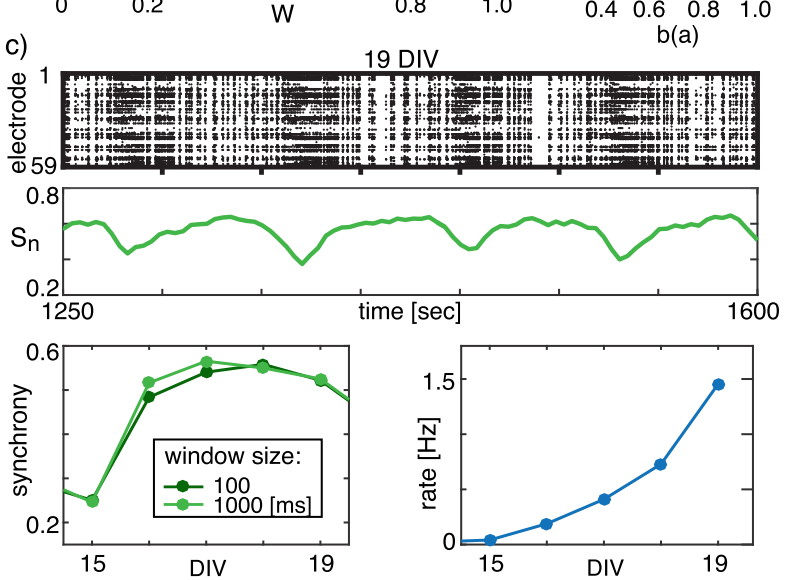

FIG. 3. (a) Schematic properties of the macroscopic model: As parameter $W$ is increased, the stability of the limit cycle (blue) decreases (Lyapunov exponent $\lambda \lesssim 0$ ), while the fixed point (red) becomes less repelling [real part of c.c. eigenvalues $R\left(\epsilon_{1,2}\right) \gtrsim 0$, middle]. Due to intrinsic fluctuations, the system spends more time in a desynchronized state, corresponding to the convex hump in the synchrony of Fig. 2(b). (b) Close qualitative correspondence between network behavior (left) and the macroscopic properties for linearly covarying a and $b$ (right) highlighted by the synchronization measure vs the real part of the complex conjugate eigenvalues $\epsilon_{1,2}$ at $\left\{x^{*}, y^{*}\right\}$, and by $\lambda_{1}$ vs $\lambda$, valid across the whole window of primary computational significance (shaded, cf. text). (c) In vitro neural culture exhibiting the characteristic behavior around point $C$ of Fig. 2: Time-resolved synchronous firing episodes interrupted by desynchronized bursts-maximal at day in vitro (DIV) 19 (top)-mark the emergence of maximal synchrony (middle; bottom left). Averaged electrode spike rates also follow the simulated network behavior (bottom right).

on media composition, which may explain the lower experimental rates.

\section{A. Experimental data preparation}

Neural culture activity data were recorded and provided by Professor Yoonkey Nam's group at the Korea Advanced Institute of 
Science and Technology (KAIST), in accordance with the approval of the Institutional Animal Care and Use Committee (IACUC). Detailed discussion of the experimental procedures can be found in Refs. 31 and 33. Essential details are as follows: Neural spiking activity was recorded from dissociated Sprague-Dawley (SD) rat hippocampal neurons (Koatech, Republic of Korea). Hippocampi were microsurgically separated from the embryonic day 18 rat, the dissected tissue was dissociated using Hank's buffer salt solution, and cells were plated on a multielectrode array (MEA) chip at a density of 400 cells $/ \mathrm{mm}^{2}$. Neural spiking activity was recorded using MEA with 59 microelectrodes (TiN, $30 \mu \mathrm{m}$ in diameter, $200 \mu \mathrm{m}$ in spacing) and one reference electrode (Multi Channel Systems, Reutlingen, Germany). Spikes were detected by setting the threshold level at six times the standard deviation of the background noise.

\section{CONCLUSION AND OUTLOOK}

In conclusion, we have shown that close-to-biology models of cortical (mini)columns exhibit, upon a neurotransmitterlike increase of the synaptic strength, universal firing changes. For values of synaptic strength $W$ where avalanche size or duration appear to follow power laws, the largest Lyapunov exponent remains positive (supplementary material). Our revealed firing paradigm is exceptionally stable against changes in network topology, rewiring, and neural dynamics. This indicates that the recently found nonequivalence ${ }^{13}$ between the two commonly used notions of criticality for networks with node dynamics ("avalanche" and "edgeof-chaos" criticality ${ }^{34}$ ) may be more generic than exceptional (at least in the early developmental phase of neural networks). Moreover, the central aspects of the observed firing transitions and their robustness could be explained by a macroscopic model. Finally, we provided supporting evidence for the modality changes and their generic nature from in vitro experiments of dissociated neuron cultures.

The simple and fundamental nature of our macroscopic model's resource-activity dynamics suggests that the revealed paradigm might be pivotal for getting a grip on other seemingly different neural circuits as well, such as predominantly GABAergic (inhibitory) networks, ${ }^{35}$ e.g., the mammalian circadian pacemaker with the suprachiasmatic nucleus, ${ }^{36}$ which is also known to exhibit counterintuitive synchronization phenomena with respect to changes in coupling. ${ }^{37,38}$

Previous efforts to link realistic neuron firing patterns with properties of dynamical stability were either based on probabilistic binary ${ }^{39}$ or on abstract "rate" neurons. ${ }^{40}$ Our work completes these studies at a more biologically detailed implementation level. The modeled network class still leaves a number of properties characterizing fully realistic in vivo neural network behavior (e.g., growing networks with variable neuron classes, facilitation and depression of connections on intermediate time scales) unconsidered. The remarkable stability of the paradigm within the wide class of neural networks studied indicates, however, that an abstract nonlinear dynamics analysis similar to the one provided here will explain the firing behavior in these cases as well. This is even more so since for a following phase of the development, where these additional features need to be included in the modeling, similar, albeit slightly more complicated, universality principles emerge (to be published). This remarkable fact suggests that superordinated parts in large and complex neural systems could, at each excitation level, rely on receiving statistically narrowly characterized firing patterns that are independent of the precise wiring of the generating modules. For understanding the brain, this provokes the question to what degree details below the columnar dimension could be relevant and conversely raises the expectation that, perhaps, much of the overall dynamics of biological neural networks is effectively explained by relatively simple models of neuronal dynamics (e.g., generalized Kuramoto models ${ }^{41,42}$ ). For the moment, a comparison of our results with the standard Kuramoto models suggests that the paradigm discovered here is different in various respects: not only that our contributing elements are chaotic, but also the organization of synchronization seems to follow a different organization scheme.

\section{SUPPLEMENTARY MATERIAL}

Our additional "supplementary material” contains Sec. I reporting the details of the construction of the investigated network types [random networks (RA), small-world networks (SW), 3D columnar topology (CL), and layered 3D columnar topology (LCL)], demonstrating that a vast scope of network types were checked. In Sec. II, we report details of our tests regarding the robustness of the reported firing paradigm [effect of randomization of neural parameters and network topology, effect of the in-degree constraint on networks, effects of resampling parameters and network topology, effects of persistently firing neurons (PN), effects of layered canonical columnar network structure-all of which we show to be negligible]. Section III contains details regarding our meanfieldlike model, followed by Sec. IV detailing our Lyapunov spectra and KS-entropy calculation methods. In Sec. V, we provide a more detailed description of the observed firing paradigm, followed by Sec. VI on how we verified the occurrence of avalanche criticality. The final section exposes the physics origins of the concept of criticality.

\section{ACKNOWLEDGMENTS}

The authors acknowledge the support by the Swiss National Science Foundation (Grant No. 200021 153542/1), by ETH (Grant No. ETH-37 152), and by the Swiss-Korea Collaboration (Grant No. IZKS2 162190).

\section{REFERENCES}

${ }^{1}$ M. Barahona and L. M. Pecora, "Synchronization in small-world systems," Phys. Rev. Lett. 89(5), 054101 (2002).

${ }^{2}$ E. Niebur, H. G Schuster, D. M Kammen, and C. Koch, "Oscillator-phase coupling for different two-dimensional network connectivities,” Phys. Rev. A 44(10), 6895 (1991).

${ }^{3}$ C. Zhou, A. E Motter, and J. Kurths, "Universality in the synchronization of weighted random networks," Phys. Rev. Lett. 96(3), 034101 (2006).

${ }^{4}$ D. Hansel and C. van Vreeswijk, "The mechanism of orientation selectivity in primary visual cortex without a functional map," J. Neurosci. 32(12), 4049 (2012). ${ }^{5}$ R. Stoop, V. Saase, C. Wagner, B. Stoop, and R. Stoop, "Beyond scale-free smallworld networks: Cortical columns for quick brains," Phys. Rev. Lett. 110(10), 108105 (2013).

${ }^{6}$ A. A Prinz, D. Bucher, and E. Marder, "Similar network activity from disparate circuit parameters,” Nat. Neurosci. 7(12), 1345 (2004).

${ }^{7}$ T. P. Vogels, K. Rajan, and L. F. Abbott, "Neural network dynamics," Annu. Rev. Neurosci. 28, 357 (2005). 
${ }^{8} \mathrm{H}$. Markram et al., "Reconstruction and simulation of neocortical microcircuitry," Cell 163(2), 456 (2015).

${ }^{9}$ C. I. Bargmann and E. Marder, "From the connectome to brain function," Nat. Methods 10(6), 483 (2013)

${ }^{10} \mathrm{C}$. M. Constantinople and R. M. Bruno, "Effects and mechanisms of wakefulness on local cortical networks," Neuron 69(6), 1061 (2011).

${ }^{11}$ P. Dayan, "Twenty-five lessons from computational neuromodulation," Neuron 76(1), 240-256 (2012).

${ }^{12}$ S.-H. Lee and Y. Dan, "Neuromodulation of brain states," Neuron 76(1), 209-222 (2012).

${ }^{13} \mathrm{~K}$. Kanders, T. Lorimer, and R. Stoop, "Avalanche and edge-of-chaos criticality do not necessarily co-occur in neural networks," Chaos 27(4), 047408 (2017).

${ }^{14}$ Z. Molnár, "Cortical columns," in Neural Circuit Development and Function in the Brain (Elsevier, 2013), pp. 109-129.

${ }^{15} \mathrm{~B}$. Roerig and B. Chen, "Relationships of local inhibitory and excitatory circuits to orientation preference maps in ferret visual cortex," Cerebr. Cortex 12, 187198 (2002).

${ }^{16}$ S. Song, P. J. Sjöström, M. Reigl, S. Nelson, and D. B. Chklovskii, "Highly nonrandom features of synaptic connectivity in local cortical circuits," PLoS Biol. 3(3), e68 (2005).

${ }^{17}$ S. H. Hendry, H. D. Schwark, E. G. Jones, and J. Yan, "Numbers and proportions of GABA-immunoreactive neurons in different areas of monkey cerebral cortex," Teurosci. 7(5), 1503-1519 (1987).

${ }^{18} \mathrm{~N}$. F. Rulkov, "Modeling of spiking-bursting neural behavior using twodimensional map," Phys. Rev. E 65(4), 041922 (2002).

${ }^{19}$ N. F. Rulkov, I. Timofeev, and M. Bazhenov, "Oscillations in large-scale cortical networks: Map-based model," J. Comput. Neurosci. 17(2), 203-223 (2004).

${ }^{20} \mathrm{~K}$. Kanders and R. Stoop, "Phase response properties of Rulkov model neurons," in Emergent Complexity from Nonlinearity, in Physics, Engineering and the Life Sciences, Springer Proceedings in Physics (Springer, 2017), pp. 21-35.

${ }^{21}$ P. Fatt and B. Katz, "Some observations on biological noise," Nature 166(4223), 597 (1950).

${ }^{22}$ S. A. Prescott, Y. De Koninck, and T. J. Sejnowski, "Biophysical basis for three distinct dynamical mechanisms of action potential initiation," PLoS Comput. Biol. 4(10), e1000198 (2008).

${ }^{23}$ A. V. Egorov, B. N. Hamam, E. Fransén, M. E. Hasselmo, and A. A. Alonso, "Graded persistent activity in entorhinal cortex neurons," Nature 420(6912), 173 (2002).

${ }^{24}$ J.-P. Eckmann, S. Jacobi, S. Marom, E. Moses, and C. Zbinden, "Leader neurons in population bursts of 2D living neural networks," New J. Phys. 10(1), 015011 (2008).

${ }^{25}$ J. G. Orlandi, J. Soriano, E. Alvarez-Lacalle, S. Teller, and J. Casademunt, "Noise focusing and the emergence of coherent activity in neuronal cultures," Nat. Phys. 9(9), 582 (2013).

${ }^{26} \mathrm{D}$. Golomb and J. Rinzel, "Clustering in globally coupled inhibitory neurons," Physica D 72(3), 259-282 (1994).
${ }^{27}$ J. M. Beggs and D. Plenz, "Neuronal avalanches in neocortical circuits," J. Neurosci. 23(35), 11167-11177 (2003).

${ }^{28}$ J. Peinke, J. Parisi, O. E. Rössler, and R. Stoop, Encounter with Chaos: Self-Organized Hierarchical Complexity in Semiconductor Experiments (Springer Science \& Business Media, 2012).

${ }^{29} \mathrm{~J}$. Hofbauer and K. Sigmund, The Theory of Evolution and Dynamical Systems: Mathematical Aspects of Selection (Cambridge University Press, 1988).

${ }^{30} \mathrm{M}$. Ichikawa, K. Muramoto, K. Kobayashi, M. Kawahara, and Y. Kuroda, "Formation and maturation of synapses in primary cultures of rat cerebral cortical cells: An electron microscopic study," Neurosci. Res. 16(2), 95-103 (1993).

${ }^{31}$ D. Berger, S. Joo, T. Lorimer, Y. Nam, and R. Stoop, "Power laws in neuronal culture activity from limited availability of a shared resource," in Emergent Complexity from Nonlinearity, in Physics, Engineering and the Life Sciences (Springer, 2017), pp. 209-220.

${ }^{32}$ R. Baddeley, L. F. Abbott, M. C. A. Booth, F. Sengpiel, T. Freeman, E. A. Wakeman, and E. T. Rolls, "Responses of neurons in primary and inferior temporal visual cortices to natural scenes," Proc. Biol. Sci. 264(1389), 1775-1783 (1997).

${ }^{33} \mathrm{~K}$. Kanders, "How topology and dynamics shape the function of neural systems," Ph.D. thesis (ETH Zürich, 2019).

${ }^{34}$ D. R. Chialvo, "Emergent complex neural dynamics," Nat. Phys. 6(10), 744 (2010).

${ }^{35} \mathrm{M}$. Bartos, I. Vida, and P. Jonas, "Synaptic mechanisms of synchronized gamma oscillations in inhibitory interneuron networks," Nat. Rev. Neurosci. 8(1), 45-56 (2007).

${ }^{36}$ E. D. Herzog, T. Hermanstyne, N. J. Smyllie, and M. H. Hastings, "Regulating the suprachiasmatic nucleus (SCN) circadian clockwork: Interplay between cellautonomous and circuit-level mechanisms," Cold Spring Harb. Perspect. Biol. 9(1), a027706 (2017).

${ }^{37}$ G. M. Freeman, Jr., R. M. Krock, S. J. Aton, P. Thaben, and E. D. Herzog, "GABA networks destabilize genetic oscillations in the circadian pacemaker," Neuron 78(5), 799-806 (2013).

${ }^{38}$ S. An, R. Harang, K. Meeker, D. Granados-Fuentes, C. A. Tsai, C. Mazuski, J. Kim, F. J. Doyle III, L. R. Petzold, and E. D. Herzog, "A neuropeptide speeds circadian entrainment by reducing intercellular synchrony," Proc. Natl. Acad. Sci. U.S.A. 110, E4355-E4361 (2013).

${ }^{39} \mathrm{C}$. Haldeman and J. M. Beggs, "Critical branching captures activity in living neural networks and maximizes the number of metastable states," Phys. Rev. Lett. 94(5), 058101 (2005).

${ }^{40}$ M. O. Magnasco, O. Piro, and G. A. Cecchi, "Self-tuned critical anti-Hebbian networks," Phys. Rev. Lett. 102(25), 258102 (2009).

${ }^{41}$ A. Pikovsky and M. Rosenblum, "Dynamics of globally coupled oscillators: Progress and perspectives," Chaos 25(9), 097616 (2015).

${ }^{42} \mathrm{M}$. Rosenblum and R. Cestnik, "Reconstructing networks of pulse-coupled oscillators from spike trains," Phys. Rev. E 96(1), 012209 (2017). 